\title{
A meta-analysis to determine the efficacy and tolerability of anti-B-cell monoclonal antibodies in multiple sclerosis
}

\author{
QINFANG XIE, XIAOLING LI, JINGJIE SUN, BOYAO YUAN, YIJUN LI, LIJUAN WANG and MANXIA WANG
}

Department of Neurology, Lanzhou University Second Hospital, Lanzhou, Gansu 730030, P.R. China

Received July 30, 2015; Accepted December 19, 2016

DOI: 10.3892/etm.2017.4298

\begin{abstract}
The present study performed a meta-analysis of randomized controlled trials in multiple sclerosis (MS) patients to evaluate the efficacy and safety of anti-B-cell monoclonal antibodies (mAbs). To the best of our knowledge, no previous meta-analysis has evaluated this. Relevant studies published until March 2015 were retrieved from the PubMed, EMBASE and Cochrane Library using the following keywords: 'Clinical trial', 'randomized', 'multiple sclerosis' or 'MS' and 'monoclonal antibodies' or 'mAbs'. Two authors independently selected the articles and extracted the data. The meta-analysis was performed using Review Manager version 5.3 software. Four randomized clinical trials comprising a total of 745 patients were selected. Anti-B-cell mAb treatment reduced the formation of gadolinium-enhancing lesions [mean difference $(\mathrm{MD})=-5.62 ; 95 \%$ confidence interval $(\mathrm{CI})=-8.00$ to -3.24 ; $\mathrm{P}<0.001)$ and was associated with smaller volume changes of lesions on T2-weighted magnetic resonance imaging ( $\mathrm{MD}=-604.40 ; 95 \% \mathrm{CI}=-941.23$ to -267.57 ; $\mathrm{P}<0.001$ ). It also significantly reduced the proportion of MS patients having at least one relapse [odds ratio $(\mathrm{OR})=0.25 ; 95 \% \mathrm{CI}=0.14-0.44$; $\mathrm{P}<0.001)$. Compared to placebo, anti-B-cell $\mathrm{mAb}$ treatment did not increase the frequency of adverse events $(\mathrm{OR}=0.90$; 95\% $\mathrm{CI}=0.54-1.49 ; \mathrm{P}=0.68)$ and serious adverse events $(\mathrm{OR}=1.13 ; 95 \% \mathrm{CI}=0.70-1.80 ; \mathrm{P}=0.62)$. In conclusion, the present meta-analysis suggested that anti-B-cell mAbs are a relatively effective and safe treatment for MS.
\end{abstract}

\section{Introduction}

Multiple sclerosis (MS) is an immune system-mediated inflammatory disease that is characterized by the destruction of myelin in the central nervous system (CNS). Clinical manifestations include loss of vision, extra-ocular movement disorders, paresthesias, loss of sensation, weakness, dysarthria,

Correspondence to: Professor Manxia Wang, Department of Neurology, Lanzhou University Second Hospital, 82 Cuiyingmen, Chengguan, Lanzhou, Gansu 730030, P.R. China

E-mail:wmx322@aliyun.com

Key words: monoclonal antibodies, multiple sclerosis, meta-analysis spasticity, ataxia and bladder dysfunction. Although the pathogeny of MS is still unknown, it is thought that environmental factors (e.g. vitamin D levels, Epstein-Barr virus infections and geographic latitude) as well as genetic factors have an important role (1-3).

MS places a huge burden on affected patients and on society. MS typically begins in early adulthood and is the most common neurological, non-traumatic cause of disability (4). MS is more common in women and the overall incidence rate is 3.5-6.6 per 100,000 individuals (5). The main goals of MS therapy are the prevention of relapses and disease progression. In the mid-1990s, glatiramer acetate and interferon- $\beta$ were introduced as first-line drugs. A decade later, the first oral drugs, fingolimod, dimethyl fumarate and teriflunomide, were introduced. Over the last decade, research has focused on the development of targeted therapies, and several novel drugs are now either approved, or in phase-II or -III clinical trials (6). Monoclonal antibodies (mAbs) are a class of targeted drugs. Drugs targeting the immune system are effective MS treatments, as T- and B lymphocytes may have an important role in the formation of CNS lesions (7). mAbs for use in humans are prepared using recombinant DNA technology, with the most commonly used mAbs for the treatment of MS being humanized mAbs (e.g., natalizumab, alemtuzumab or daclizumab) or mouse/human chimeric mAbs (e.g., rituximab).

The role of B cells and their products in the pathogenesis of MS has been studied for decades. Studies show that B cells may have a substantial role in MS pathogenesis and development (8). Based on these reasons, treatments that specifically target B cells were applied to MS, and CD20-targeting mAbs were developed. Rituximab, ocrelizumab and ofatumumab are all based on mAbs targeting $\mathrm{CD} 20^{+} \mathrm{B}$-cells. Several clinical trials assessing the safety and efficacy of anti-B-cell mAbs in patients with MS have already been completed or are ongoing. However, no long-term trials evaluating the efficacy and safety of anti-B-cell mAbs treatment for MS are currently available and the sample size in previous studies is small. Therefore, the present study performed a meta-analysis to evaluate the efficacy and safety of anti-B-cell mAbs in placebo-controlled, randomized clinical trials in MS patients. Meta-analysis is the statistical pooling of results from several studies in order to estimate the effects of a treatment. It overcomes the limitations of small sample size and rare outcomes, and allows for the application of powerful statistical techniques to study the effects of interest (9). 


\section{Materials and methods}

Data sources. A comprehensive and systematic literature search was performed for all randomized clinical trials that compared anti-B-cell mAbs (rituximab, ocrelizumab or ofatumumab) and a placebo in MS patients. The PubMed, EMBASE and Cochrane Library databases were searched for studies published until March 2015 using the medical subject heading terms 'clinical trial', 'randomized', 'multiple sclerosis' or 'MS' and the drug names 'monoclonal antibodies' or 'mAbs'. The reference lists of all included studies and any associated review articles were also searched to identify any relevant studies that were missed in the initial search.

Study selection. The full-length articles of studies evaluating $\mathrm{mAbs}$ and MS were selected if they fulfilled the following inclusion criteria: i) The study population was composed of MS patients with any type of MS, ii) the treatment intervention was rituximab, ocrelizumab or ofatumumab, iii) the comparison group was treated with a placebo, and iv) the trial was a randomized controlled trial. Exclusion criteria were as follows: i) The patients were not diagnosed with MS, ii) the treatment intervention was another type of $\mathrm{mAb}$, iii) the comparison group received another drug for the treatment of MS, and iv) the studies were non-randomized clinical trials. Two reviewers independently scrutinized the electronic literature searches and obtained full-length articles of all the citations that met the predefined selection criteria. Any discrepancies were solved by discussion. After identifying the articles and screening the abstracts, the studies were read in full. Ultimately, four primary studies met the inclusion criteria.

Outcomes. Lesions detected by magnetic resonance imaging (MRI) and relapse frequency are the key measures of success in MS treatment trials and are frequently used as primary outcome measures, which were adopted in the present study. The secondary outcomes were patients who experienced adverse and serious adverse events.

Data extraction. The following information was extracted from the studies: The first author's last name, year of publication, duration of treatment, sample size, gender, mean age, volume change of MS lesion from baseline on T2-weighted MRI, relapse and adverse events (Table I). Two authors extracted the data independently and any discrepancies were solved by discussion.

Statistical analysis. The meta-analysis and all statistical tests were performed and forest plots were created using RevMan 5 software, version 5.3 (Cochrane Community, London, UK). For dichotomous data, outcomes were pooled using the Mantel-Haenszel method, and measures of effect are presented as odds ratios (ORs) with 95\% confidence intervals (CIs). For continuous data, the sample size-weighted mean difference (MD) was calculated when outcomes were measured in the same manner among studies. The presence of significant heterogeneity was examined using the I squared $\left(\mathrm{I}^{2}\right)$ test, a measure of how much variance between studies can be attributed to differences between studies rather than chance. A larger $\mathrm{I}^{2}$ value indicates greater heterogeneity. In cases where

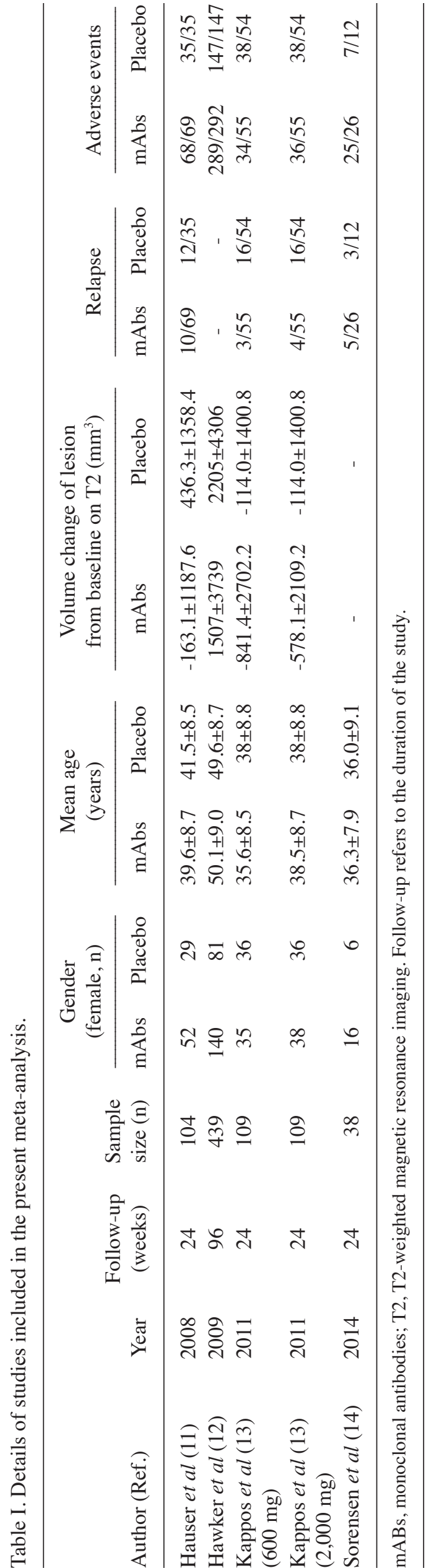




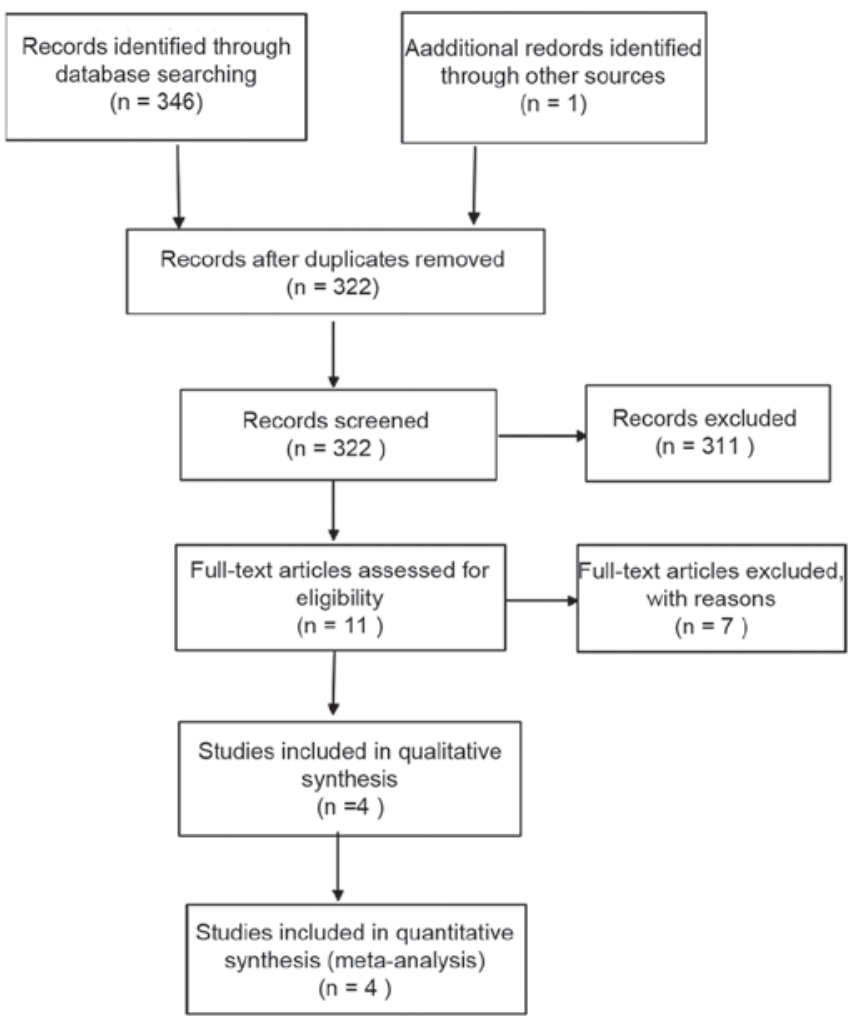

Figure 1. Flow chart illustrating the strategy of selection of studies included in the present meta-analysis.

$\mathrm{I}^{2}$ exceeded $50 \%$, results were pooled using random effects models; otherwise, fixed effects models were used. Forest plots are graphical displays that show the pooled point estimate (95\% CIs) for each outcome of interest. Forest plots were used to show the results from individual studies together with the combined result. The present meta-analysis was performed according to the Preferred Reporting Items for Systematic Reviews and Meta-Analysis statement protocol (10).

\section{Results}

Study characteristics. In the initial literature search, 346 studies were identified, 335 of which were excluded by screening of their title and abstracts. After reviewing the full text of the remaining 11 studies, four studies, including five trials (one study was regarded separately as two trials due to different dosages) with a total of 745 patients contained sufficient information to be included in the statistical analysis, and compared anti-B-cell mAbs with a placebo (11-14). The most common reasons for exclusion were that studies were reviews, contained patients not diagnosed with MS, non-randomized clinical trials, use of a mAb other than rituximab, ocrelizumab or ofatumumab as a treatment intervention, and insufficient data (Fig. 1). The risk of bias for each criterion was assessed in six components, including adequate sequence generation, allocation concealment, blinding, incomplete outcome data addressed, selective reporting and other bias by using RevMan software (Fig. 2).

Number of gadolinium-enhanced lesions. The number of gadolinium-enhanced lesions on MRI was reported by three

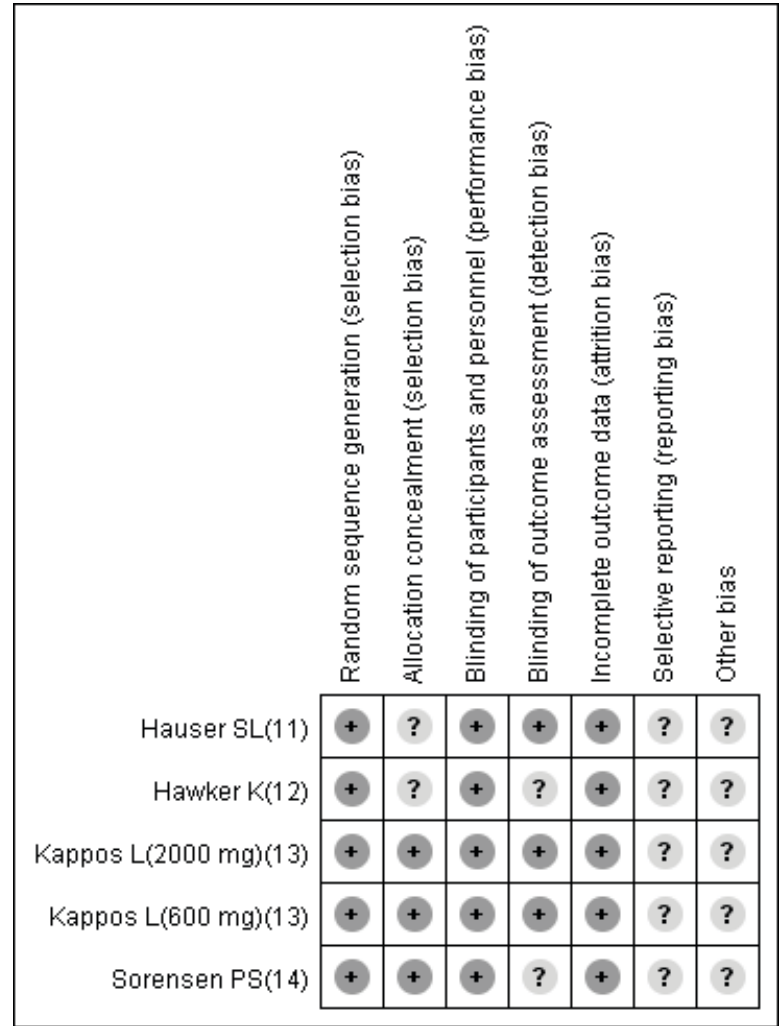

Figure 2. Risk of bias for each criterion for each included study.

trials involving 268 patients $(11,13)$. Meta-analysis showed that significantly fewer gadolinium-enhanced lesions were encountered in the anti-B-cell $\mathrm{mAbs}$ group than in the placebo group ( $\mathrm{MD}=-5.62 ; 95 \% \mathrm{CI}=-8.00$ to $-3.24 ; \mathrm{P}<0.001 ;$ Fig. 3 ). No heterogeneity existed between these studies $\left(\mathrm{I}^{2}=0 \%, \mathrm{P}<0.001\right)$.

Volume change of lesions from baseline on T2-weighted MRI. Four trials involving 707 patients provided data on the volume change of lesions from baseline on T2-weighted MRI (11-13). As presented in Fig. 4, compared with the placebo group, the anti-B-cell monoclonal antibodies group showed significantly lower changes in lesion volume on T2-weighted MRI ( $\mathrm{MD}=-604.40 ; 95 \% \mathrm{CI}=-941.23$ to $-267.57 ; \mathrm{P}<0.001)$. No heterogeneity existed between these studies $\left(\mathrm{I}^{2}=0 \%, \mathrm{P}<0.001\right)$.

Proportion of patients with relapse. Four trials involving 306 patients described the proportion of patients who relapsed $(11,13,14)$. Meta-analysis demonstrated that the proportion of patients who had at least one relapse of MS was significantly reduced by anti-B-cell $\mathrm{mAb}$ treatment $(\mathrm{OR}=0.25$; 95\% CI=0.14-0.44; $\mathrm{P}<0.001 ;$ Fig. 5 ). No heterogeneity existed between these studies $\left(\mathrm{I}^{2}=0 \%, \mathrm{P}<0.001\right)$.

Adverse events and serious adverse events. All of the five studies reported on adverse events (11-14). Adverse events were graded according to the National Cancer Institute Common Terminology Criteria for Adverse Events, version 3.0. In the overall analysis, there was no significant difference in the frequency of adverse events between the anti-B-cell $\mathrm{mAb}$ treatment groups and the placebo groups $(\mathrm{OR}=0.90 ; 95 \%$ $\mathrm{CI}=0.54-1.49 ; \mathrm{P}=0.68 ;$ Fig. 6A). Heterogeneity existed among 
Table II. Meta-analysis of other reported adverse events, excluding adverse and serious adverse events.

\begin{tabular}{|c|c|c|c|c|c|}
\hline \multirow[b]{2}{*}{ Adverse event } & \multicolumn{2}{|c|}{ Events/group size (n) } & \multirow[b]{2}{*}{ P-value } & \multirow[b]{2}{*}{ OR } & \multirow[b]{2}{*}{$95 \% \mathrm{CI}$} \\
\hline & $\mathrm{mAbs}$ & Placebo & & & \\
\hline Fatigue & $45 / 387$ & $9 / 194$ & $0.0090^{\mathrm{a}}$ & 2.68 & $1.28-5.62$ \\
\hline Headache & $149 / 497$ & $18 / 302$ & $0.0300^{\mathrm{a}}$ & 1.87 & $1.06-3.29$ \\
\hline Chills & $48 / 471$ & $4 / 290$ & $0.0002^{\mathrm{a}}$ & 5.37 & $2.19-13.16$ \\
\hline Throat irritation & $38 / 387$ & $0 / 194$ & $0.0010^{\mathrm{a}}$ & 15.93 & $3.00-84.67$ \\
\hline Infection & $298 / 497$ & $171 / 302$ & 0.9400 & 0.99 & $0.73-1.34$ \\
\hline Urinary tract infection & $15 / 179$ & $13 / 143$ & 0.6200 & 0.82 & $0.37-1.83$ \\
\hline Nasopharyngitis & $15 / 179$ & $10 / 143$ & 0.7200 & 0.85 & $0.36-2.04$ \\
\hline Upper respiratory tract infection & $23 / 205$ & $12 / 155$ & 0.6400 & 1.20 & $0.56-2.54$ \\
\hline
\end{tabular}

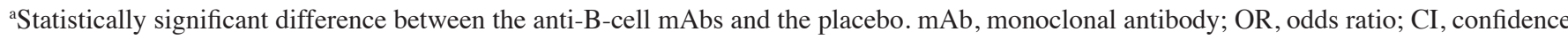
interval.

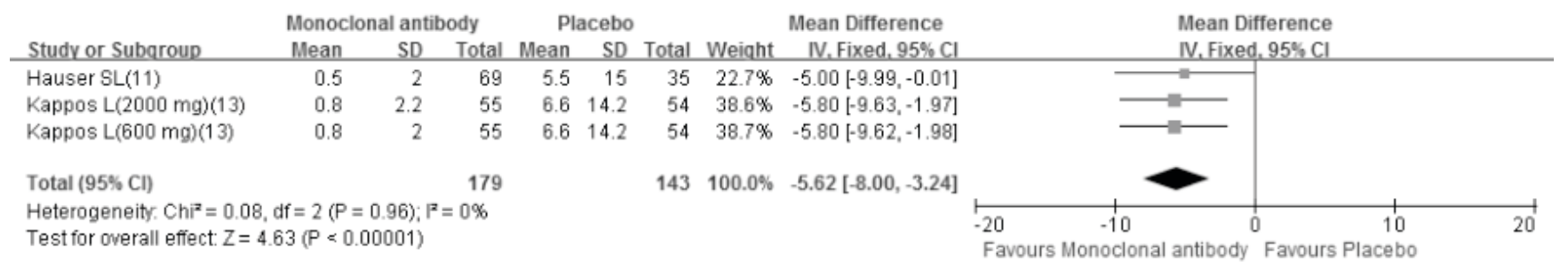

Figure 3. Forest plot showing the number of gadolinium-enhanced lesions on magnetic resonance imaging. SD, standard deviation; df, degrees of freedom; CI, confidence interval.

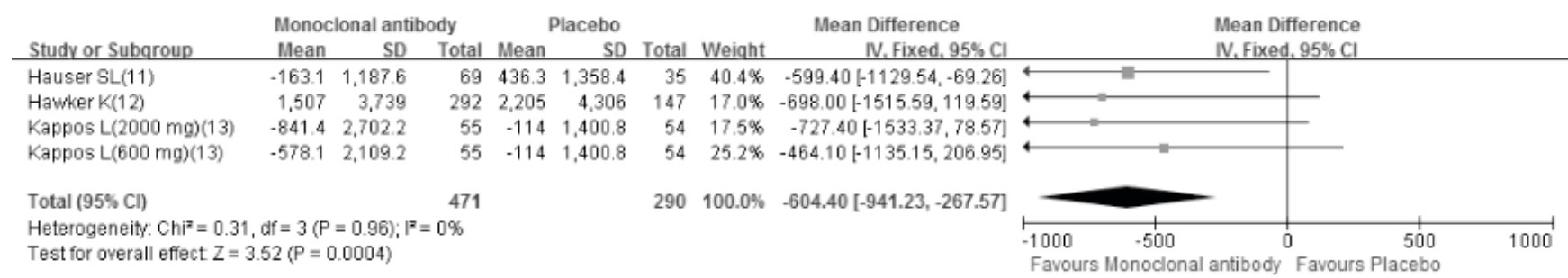

Figure 4. Forest plot showing volume changes of lesions $\left(\mathrm{mm}^{3}\right)$ from baseline on T2-weighted magnetic resonance imaging. SD, standard deviation; df, degrees of freedom; CI, confidence interval.

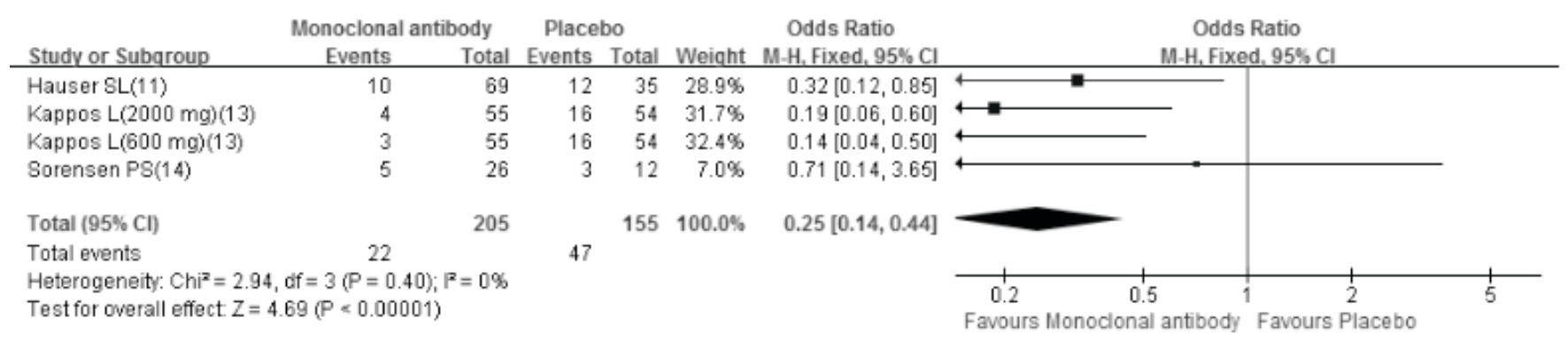

Figure 5. Forest plot showing the proportion of patients who had at least one relapse of multiple sclerosis. SD, standard deviation; df, degrees of freedom; CI, confidence interval; M-H, Mantel-Haenszel.

the included studies $\left(\mathrm{I}^{2}=48 \%, \mathrm{P}=0.68\right)$. Serious adverse events were reported in five studies (11-14). As above, the overall results of the meta-analysis revealed that patients receiving
anti-B-cell $\mathrm{mAbs}$ exhibited no significant difference in serious adverse events compared with those in the placebo group $(\mathrm{OR}=1.13 ; 95 \% \mathrm{CI}=0.70-1.80 ; \mathrm{P}=0.62$; Fig. 6B). No 


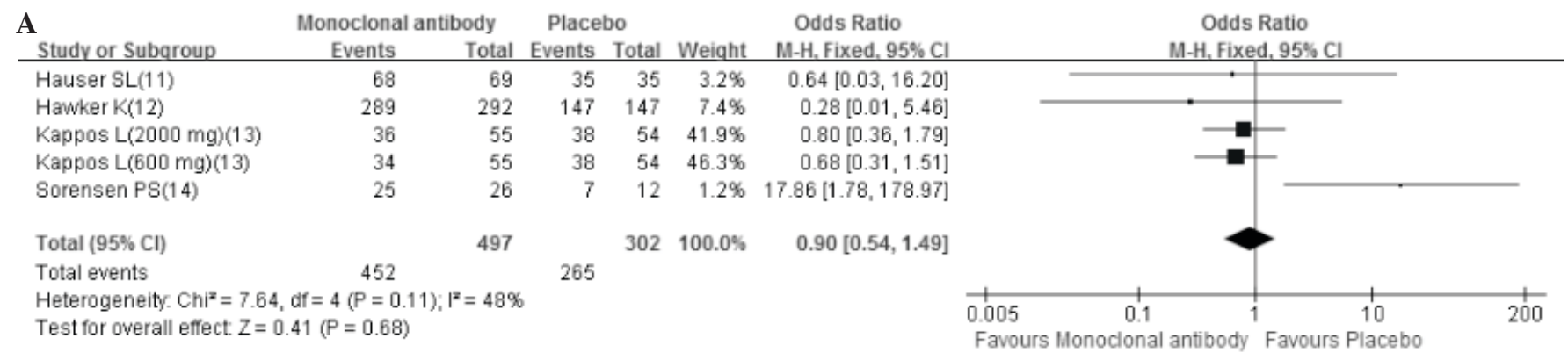

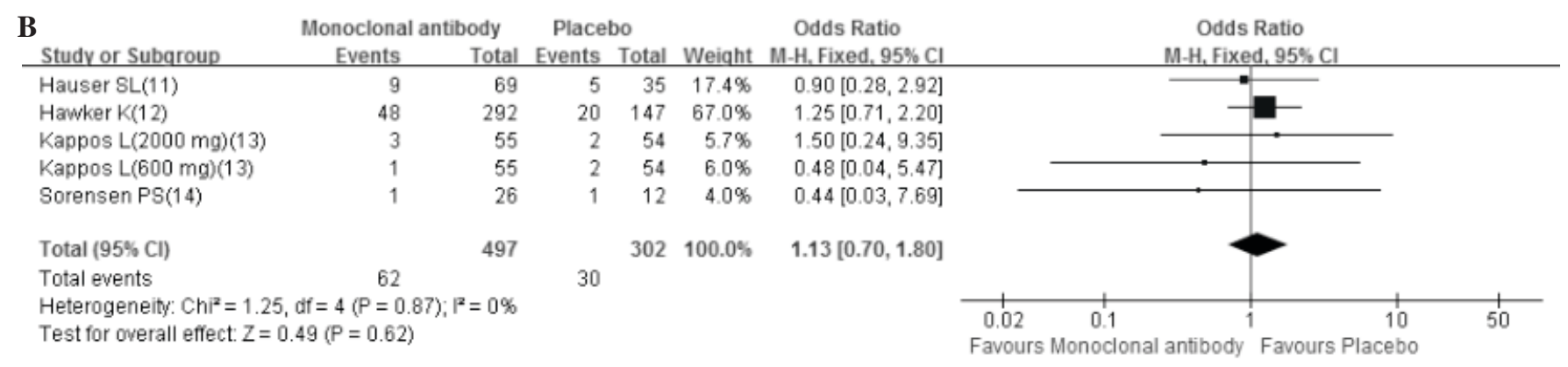

Figure 6. Forest plot showing the incidence of (A) adverse events and (B) serious adverse events. Adverse events were graded according to the National Cancer Institute Common Terminology Criteria for Adverse Events, version 3.0. SD, standard deviation; df, degrees of freedom; CI, confidence interval; M-H, Mantel-Haenszel.

heterogeneity existed between the above studies $\left(\mathrm{I}^{2}=0 \%\right.$, $\mathrm{P}=0.62$ ).

The adverse events that occurred more frequently in patients receiving anti-B-cell mAbs compared with those in patients receiving placebo were fatigue $(\mathrm{OR}=2.70 ; 95 \% \mathrm{CI}=1.29-5.65$; $\mathrm{P}=0.008)$, headache $(\mathrm{OR}=1.87 ; 95 \% \mathrm{CI}=1.06-3.29 ; \mathrm{P}=0.03)$, chills $(\mathrm{OR}=5.37 ; 95 \% \mathrm{CI}=2.19-13.16 ; \mathrm{P}<0.001)$ and throat irritation $(\mathrm{OR}=15.93$; 95\% $\mathrm{CI}=3.00-84.67$; $\mathrm{P}=0.001$; Table II). All other reported adverse events, including all infection events, urinary tract infection, nasopharyngitis and upper respiratory tract infection did not differ significantly between patients receiving anti-B-cell $\mathrm{mAbs}$ and those receiving placebo (Table II).

\section{Discussion}

To the best of our knowledge, the present meta-analysis was the first to evaluate the efficacy and tolerability of anti-B-cell mAbs in patients with MS, although a previous study performed a meta-analyses on a different type of mAb (15). The meta-analysis included studies using rituximab, ocrelizumab and ofatumumab, thus broadening the number of studies and enlarging the population size. Natalizumab, daclizumab and alemtuzumab were excluded from the present meta-analysis because they did not meet the inclusion criteria applied. Several conclusions can be drawn from the present meta-analysis. First, anti-B-cell mAbs were significantly more effective than placebo in preventing the occurrence of new gadolinium-enhanced lesions, volume changes of lesions from baseline on T2-weighted MRI and relapse of MS. Furthermore, there was no significant difference between the anti-B-cell monoclonal antibodies and placebo in terms of overall adverse events and serious adverse events. However, fatigue, headache, chills and throat irritation occurred more frequently in anti-B-cell mAb-treated groups. Importantly, most of the adverse events can be treated using standard therapies.

Rituximab is a monoclonal antibody that under current evaluation for MS treatment. It targets the B-cell surface molecule CD20 and lyses B-cells (early pro-B cells and plasma cells are spared) (16). The main adverse events described in the two trials on rituximab examined in the present study were infusion-associated reactions and infections $(11,12)$. However, infusion-associated reactions are reduced with subsequent infusions. The most common types of infection were nasopharyngitis, upper respiratory tract infection and urinary tract infection. One of the trials described two serious infection-associated adverse events (gastroenteritis and bronchitis) in the rituximab group, which were resolved without sequelae (11). There were four deaths in these two studies (two in the placebo group and two in the rituximab group), but none of them was associated with the drug (from the rituximab group: One patient had a history of brainstem lesions and aspiration, and the other one died of homicide and from the placebo group: One patient succumbed to cardiopulmonary failure and another withdrew from the study and later died from pneumonia). These outcomes showed that rituximab is safe; however, a study in rheumatoid arthritis patients treated with rituximab concluded that there is an increased risk of progressive multifocal leukoencephalopathy (PML) (17). Therefore, a larger number of patients and an increased duration of treatment and observation are required to detect side effects associated with long-term use. Furthermore, the FDA approved the use of rituximab under certain circumstances for the treatment of chronic lymphocytic leukemia (CLL), non-Hodgkin lymphoma (NHL), rheumatoid arthritis, and granulomatosis with polyangiitis and microscopic polyangiitis (18). Although it is not approved for use in MS, it is quite often used off-label (19). 
Similar to rituximab, ocrelizumab targets CD20 cells of the B-cell lineage. The most common adverse events observed in clinical trials are associated with the first infusion, and their severity is limited to mild-to-moderate. Studies on ocrelizumab in rheumatoid arthritis and lupus patients revealed increased serious opportunistic infections that led to the termination of drug use; however, opportunistic infections have not been observed in clinical trials on MS. However, in one trial (13), one patient in the $2,000 \mathrm{mg}$ group died in week 14 due to brain edema, after the occurrence of systemic inflammatory response syndrome. Whether ocrelizumab contributed to the patient's death remains elusive. Trials over longer periods of time with large populations are required to evaluate the risk of rare adverse events such as serious opportunistic infections. No cases of PML have been reported in MS patients who received ocrelizumab treatment.

Ofatumumab is also a humanized $\mathrm{mAb}$ that targets CD20 on B lymphocytes. In the respective trial (14), the most common adverse event was an infusion-associated reaction; most adverse reactions were mild and were common on the first day of ofatumumab dosing, but were not observed on the second day of infusion. The percentage of patients with infection was similar between the mAb and placebo groups and most infections were mild or moderate. Serious adverse events were observed in 2 patients: One case in the placebo group had influenza and one case in the ofatumumab group had a headache leading to hospitalization but was considered unrelated to ofatumumab; the adverse events were resolved in the two cases. No cases of opportunistic infections and PML have been reported in association with ofatumumab treatment.

Since the present study depended on other studies, it had certain limitations. Due to the use of electronic databases and the search strategy applied, certain articles may have been missed. There may have also been bias in case selection. In addition, certain studies were included that had different baseline characteristics, including the follow-up time and mean age. Finally, only few long-term trials were available, which reduced the chance of encountering adverse events. Therefore, more log-term trials are required to assess adverse effects of treatment.

In conclusion, the results of the present meta-analysis showed that, compared to placebo treatment, the three anti-B-cell mAbs treatment has a beneficial effect and tolerable adverse events in MS patients. B-cell-targeting $\mathrm{mAb}$ treatment significantly reduced the occurrence of new gadolinium-enhanced lesions, volume changes of lesion from baseline on T2-weighted MRI and relapse. Thus, their effectiveness and tolerable safety profile make anti-B-cell mAbs desirable drugs for MS treatment. Although interferon- $\beta$ and glatiramer acetate have been used for the treatment of MS for decades and show a favorable safety profile, they can only partially protect against relapses. Natalizumab showed greater effectiveness, but carries a risk of PML. Several therapies have no available long-term safety profiles. Based on the above reasons, anti-B-cell mAbs may be promising drugs for the treatment of MS with greater efficacy and safety. Although this novel therapy is likely to have Ab-specific risks and complications, pharmacovigilance or risk management plans may make these effective treatments accessible to patients. Therefore, vigilant monitoring of patients undergoing therapy is required and long-term studies of anti-B-cell mAbs should be performed.

\section{References}

1. International Multiple Sclerosis Genetics Consortium; Wellcome Trust Case Control Consortium, Sawcer S, Hellenthal G, Pirinen M, Spencer CC, Patsopoulos NA, Moutsianas L, Dilthey A, Su Z, et al: Genetic risk and a primary role for cell-mediated immune mechanisms in multiple sclerosis. Nature 476: 214-219, 2011.

2. Ascherio A and Munger KL: Environmental risk factors for multiple sclerosis. Part I: The role of infection. Ann Neurol 61: 288-299, 2007.

3. Ascherio A and Munger KL: Environmental risk factors for multiple sclerosis. Part II: Noninfectious factors. Ann Neurol 61: 504-513, 2007.

4. Tanasescu R, Ionete C, Chou IJ and Constantinescu CS: Advances in the treatment of relapsing- Remitting multiple sclerosis. Biomed J 37: 41-49, 2014.

5. Alonso A and Hernán MA: Temporal trends in the incidence of multiple sclerosis: A systematic review. Neurology 71: 129-135, 2008.

6. Jeffery DR: Recent advances in treating multiple sclerosis: Efficacy, risks and place in therapy. Ther Adv Chronic Dis 4: 45-51, 2013.

7. Hemmer B, Archelos JJ and Hartung HP: New concepts in the immunopathogenesis of multiple sclerosis. Nat Rev Neurosci 3: 291-301, 2002.

8. Disanto G, Morahan JM, Barnett MH, Giovannoni G and Ramagopalan SV: The evidence for a role of B cells in multiple sclerosis. Neurology 78: 823-832, 2012.

9. Nordmann AJ, Kasendaa B and Briel M: Meta-analyses: What they can and cannot do. Swiss Med Wkly 142: w13518, 2012.

10. Moher D, Liberati A, Tetzlaff J, Altman DG; PRISMA Group: Preferred reporting items for systematic reviews and meta-analysis: The PRISMA statement. Ann Intern Med 151: 264-269, 2009.

11. Hauser SL, Waubant E, Arnold DL, Vollmer T, Antel J, Fox RJ, Bar-Or A, Panzara M, Sarkar N, Agarwal S, et al: B-cell depletion with rituximab in relapsing-remitting multiple sclerosis. $\mathrm{N}$ Engl J Med 358: 676-688, 2008.

12. Hawker K, O'Connor P, Freedman MS, Calabresi PA, Antel J, Simon J, Hauser S, Waubant E, Vollmer T, Panitch H, et al: Rituximab in patients with primary progressive multiple sclerosis: Results of a randomized double-blind placebo-controlled multicenter trial. Ann Neurol 66: 460-471, 2009.

13. Kappos L, Li D, Calabresi PA, O'Connor P, Bar-Or A, Barkhof F, Yin M, Leppert D, Glanzman R, Tinbergen J and Hauser SL: Ocrelizumab in relapsing-remitting multiple sclerosis: A phase 2, randomised, placebo-controlled, multicentre trial. Lancet 378: 1779-1787, 2011 .

14. Sorensen PS, Lisby S, Grove R, Derosier F, Shackelford S, Havrdova E, Drulovic J and Filippi M: Safety and efficacy of ofatumumab in relapsing-remitting multiple sclerosis: A phase 2 study. Neurology 82: 573-581, 2014.

15. Nikfar S, Rahimi R, Rezaie A and Abdollahi M: A meta-analysis on the efficacy and tolerability of natalizumab in relapsing multiple sclerosis. Arch Med Sci 6: 236-244, 2010.

16. Longbrake EE, Parks BJ and Cross AH: Monoclonal antibodies as disease modifying therapy in multiple sclerosis. Curr Neurol Neurosci Rep 13: 390, 2013.

17. Clifford DB, Ances B, Costello C, Rosen-Schmidt S, Andersson M, Parks D, Perry A, Yerra R, Schmidt R, Alvarez E and Tyler KL: Rituximab-associated progressive multifocal leukoencephalopathy in rheumatoid arthritis. Arch Neurol 68: 1156-1164, 2011.

18. US Food and Drug Administration: Rituximab (marketed as Rituxan) information. http://www.fda.gov/Drugs/DrugSafety/ PostmarketDrugSafetyInformationforPatientsandProviders/ ucm109106.htm.Accessed July 7, 2013.

19. Tony HP, Burmester G, Schulze-Koops H, Grunke M, Henes J, Kötter I, Haas J, Unger L, Lovric S, Haubitz M, et al: Safety and clinical outcomes of rituximab therapy in patients with different autoimmune diseases: Experience from a national registry (GRAID). Arthritis Res Ther 13: R75, 2011. 